\title{
Volatility Leveraging in Heart Rate: health vs disease
}

\author{
Ana Paula Rocha ${ }^{1}$, Argentina Leite ${ }^{2}$, Maria Eduarda Silva $^{3}$ \\ ${ }^{1}$ CMUP \& Faculdade de Ciências, Universidade do Porto, Porto, Portugal \\ ${ }^{2}$ C-BER \& INESC TEC \& Escola de Ciências e Tecnologia, Universidade de Trás-os-Montes e Alto \\ Douro, Vila Real, Portugal \\ ${ }^{3}$ CIDMA \& Faculdade de Economia, Universidade do Porto, Porto, Portugal
}

\begin{abstract}
Heart Rate Variability (HRV) data exhibit long memory and time-varying conditional variance (volatility). These characteristics are well captured using Fractionally Integrated AutoRegressive Moving Average (ARFIMA) models with Generalised AutoRegressive Conditional Heteroscedastic (GARCH) errors, which are an extension of the AR models usual in the analysis of HRV. GARCH models assume that volatility depends only on the magnitude of the shocks and not on their sign, meaning that positive and negative shocks have a symmetric effect on volatility. However, HRV recordings indicate further dependence of volatility on the lagged shocks. This work considers Exponential GARCH (EGARCH) models which assume that positive and negative shocks have an asymmetric effect (leverage effect) on the volatility, thus better copping with complex characteristics of HRV. ARFIMA-EGARCH models, combined with adaptive segmentation, are applied to $24 \mathrm{~h} \mathrm{HRV}$ recordings of 30 subjects from the Noltisalis database: 10 healthy, 10 patients suffering from congestive heart failure and 10 heart transplanted patients. Overall, the results for the leverage parameter indicate that volatility responds asymmetrically to values of HRV under and over the mean. Moreover, decreased leverage parameter values for sick subjects, suggest that these models allow to discriminate between the different groups.
\end{abstract}

\section{Introduction}

Heart Rate Variability (HRV) reflects the interaction between perturbations to the cardiovascular variables and the corresponding response of the cardiovascular regulatory systems [1]. The modeling of such variability can provide a quantitative and non-invasive method to assess the integrity of the cardiovascular system. HRV data display non stationarity and exhibit long memory and time-varying conditional variance (usually designated by volatility) among other nonlinear characteristics [2], that are well modelled by AutoRegressive Fractionally Integrated Moving Average (ARFIMA) models with Generalised AutoRegressive Conditional Heteroscedastic (GARCH) errors [3]. GARCH models assume that volatility depends only on the magnitude of the shocks and not on their sign, meaning that positive and negative shocks have a symmetric effect on volatility [4]. In [5] the authors consider an extension of GARCH models, Exponential GARCH (EGARCH) models, which allow for an asymmetric effect (leverage effect) and conclude that a model with leverage effect is more suited to describe the complex and nonlinear characteristics of HRV.

\section{Data and Methods}

\subsection{Data}

This study analyses HRV data from the Noltisalis database [6] which was collected by the cooperative effort of university departments and rehabilitation clinics in Italy. The dataset consists of 24 hour HRV recordings of 30 subjects: 10 healthy subjects $(\mathrm{N}, 22.5 \pm 1.6$ hours; $102115.2 \pm 11365.4$ beats; $42.2 \pm 6.4$ years), 10 patients suffering from congestive heart failure $(\mathrm{C}, 22.4 \pm 0.9$ hours; $107170.5 \pm 16689.3$ beats; $53.6 \pm 11.2$ years) and 10 heart transplanted patients (T, $22.4 \pm 0.7$ hours; $116043.3 \pm 11913.2$ beats; $44.9 \pm 14.8$ years). The starting time of the Holter diary is available, enabling to distinguish between day and night periods. Therefore, the 24 hour HRV series are analysed in three periods: 6 hours during day, 6 hours during night and 24 hours.

\subsection{ARFIMA-EGARCH modeling of $\mathrm{HRV}$}

The model considered in this work is the $\operatorname{ARFIMA}(p, d, 0)$ $\operatorname{EGARCH}(1,1)$ defined by the following equations [5]

$$
\begin{gathered}
\phi(B)(1-B)^{d} x_{t}=\epsilon_{t} \\
\epsilon_{t}=\sigma_{t} z_{t}
\end{gathered}
$$




$$
\log \sigma_{t}^{2}=u_{0}^{*}+v_{1} \log \sigma_{t-1}^{2}+u_{1}\left|z_{t-1}\right|+\xi_{1} z_{t-1}
$$

where $u_{0}^{*}=u_{0}-u_{1} \sqrt{\frac{2}{\pi}}, z_{t}=\epsilon_{t} / \sigma_{t}$ and $B$ is the backward-shift operator.

Equation (1) describes the conditional mean of the process with serially uncorrelated residuals $\epsilon_{t}$ and is said an $\operatorname{ARFIMA}(p, d, 0)$ with $p \in N_{0}$ and $d \in R$; $d$, the longmemory parameter, determines the long-term behaviour in mean and $(1-B)^{d}=\sum_{k=0}^{\infty}\left(\begin{array}{l}d \\ k\end{array}\right)(-1)^{k} B^{k}$ is the fractional difference operator [7]; and the polynomial $\phi(B)=$ $1-\phi_{1} B-\ldots-\phi_{p} B^{p}$ allows for the modeling of the shortrange properties in the mean; for $-0.5<d<0.5$ the ARFIMA process is covariance stationary. In the range $-0.5<d<0.5$, the long memory parameter is related to the Hurst coefficient, $H$, to the fractal dimension, $D$, and to the slope of the (generalised) spectral density in the low frequency range, $\alpha$, by $d=H-0.5, H=2-D$ and $\alpha=2 d$, respectively. Moreover, for $0.5 \leq d<1$ the ARFIMA process is non-stationary and mean reverting.

Equations (2) and (3) describe the conditional variance of the process which varies over time as in time-varying AR models. In (2) $\epsilon_{t}$ are called shocks and $z_{t}$, independent and identically distributed random variables with zero mean and unit variance, are the standardised shocks. This process does not require constraints on the parameters for ensuring the positivity of the variance. The parameters $u_{1}$ and $v_{1}$ characterise the volatility clustering phenomena and the parameter $\xi_{1}$ describes the leverage effect. The impact of positive shocks, $\epsilon_{t-1}>0$ is $\left(\xi_{1}+u_{1}\right) \frac{\epsilon_{t-1}}{\sigma_{t-1}}$, while for negative shocks it is $\left(\xi_{1}-u_{1}\right) \frac{\epsilon_{t-1}}{\sigma_{t-1}}$. If $\xi_{1}=0, \log \sigma_{t}^{2}$ responds symmetrically to $\epsilon_{t-1}$.

Specifically consider the tachogram of the healthy subject N5 (segment of RR series with 1300 beats) represented in Figure 1(a) to which an $\operatorname{ARFIMA}(p=6, d=$ $0.45,0)$ is fitted ${ }^{1}$. The corresponding residuals are represented in Figure 1(b). The squared residuals exhibit significant autocorrelation (ACF), Figure 1(c). Additionally, the cross-correlation (CCF) between the residuals $\hat{\epsilon}_{t}$ and their squares $\hat{\epsilon}_{t}^{2}$, Figure 1(d), indicate that the conditional variance depends also on the lagged shocks (leverage effect). Fitting an $\operatorname{EGARCH}(1,1)$ model using maximum likelihood (Econometrics Toolbox of Matlab [8]) the estimated parameters are $\hat{u}_{0}=-2.98, \hat{u}_{1}=0.23, \hat{v}_{1}=0.66$ and $\hat{\xi}_{1}=0.35$. The estimated volatility equation (3) is

$\log \sigma_{t}^{2}=-3.16+0.66 \log \sigma_{t-1}^{2}+\left\{\begin{array}{l}0.58 z_{t-1} \text { if } z_{t-1} \geq 0 \\ 0.12 z_{t-1} \text { if } z_{t-1}<0 .\end{array}\right.$

${ }^{1}$ (i) Estimate $d$ using the semi-parametric local Whittle estimator; (ii) define the filtered data $y_{t}=(1-B)^{d} x_{t}$; (iii) estimate the $\operatorname{AR}(p)$ parameters in the filtered data $y_{t}$ by maximum likelihood, with the order $p$ determined by the Akaike Information Criterion (AIC)
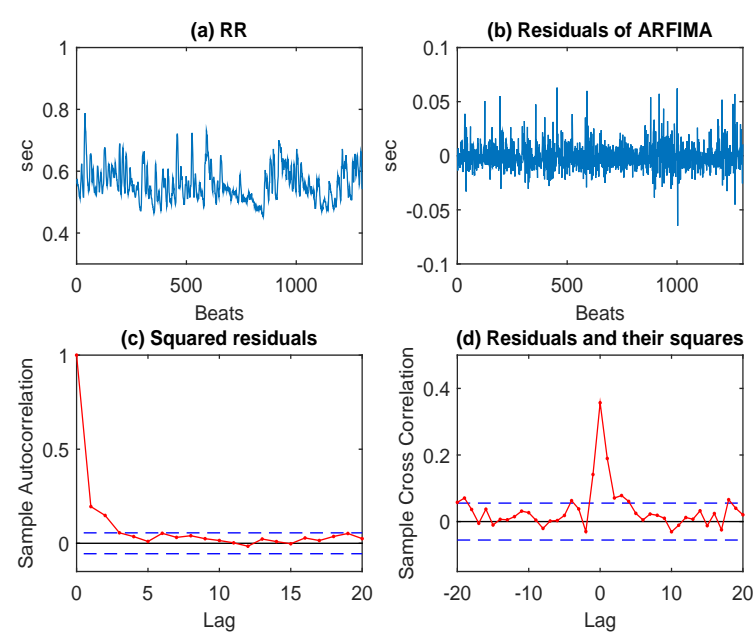

Figure 1. Short-term HRV data: (a) tachogram of a normal subject (segment with 1300 beats of RR series for subjectN5 from the Noltisalis database), (b) residuals of the fitted ARFIMA(6,0.45,0) model, (c) ACF of the squared residuals and (d) CCF of the residuals and the squared residuals. The horizontal lines (- -) show the $95 \%$ confidence limits.

Taking antilog transformation, we have

$$
\sigma_{t}^{2}=\sigma_{t-1}^{2 * 0.66} \mathrm{e}^{-3.16}\left\{\begin{array}{l}
\mathrm{e}^{0.58 z_{t-1}} \text { if } z_{t-1} \geq 0 \\
\mathrm{e}^{0.12 z_{t-1}} \text { if } z_{t-1}<0
\end{array}\right.
$$

For example, for a standardised shock with magnitude 2, we have a Shock Impact [4] of $\frac{\sigma_{t}^{2}\left(z_{t-1}=-2\right)}{\sigma_{t}^{2}\left(z_{t-1}=2\right)}=0.25$. Therefore, the impact of negative shock of size 2 standard deviations is about $75 \%$ below than that of a positive shock of the same size. Similar results were obtained in other cases.

\section{Results and discussion}

The description of 24 hour HRV recordings which are long (approximately 100000 beats) and exhibit several non stationary characteristics with circadian variation, is achieved by ARFIMA-EGARCH modeling combined with adaptive segmentation [3]: long records are decomposed into short records of variable length ( $\geq 512$ beats) and the break points, which mark the end of consecutive short records, are identified by AIC criterion. A detailed description of the ARFIMA-EGARCH modeling procedure can be found in [5].

The results are illustrated for the subjects N5, C5 and T3 in Figures 2, 3 and 4. The long memory estimates $\hat{d}$ in (b) change over time showing circadian variation, with lowest values during the night periods, $0<\hat{d}<0.5$ in contrast with $0.5<\hat{d}<1$ for the day period. The volatility parameters estimates $\hat{u}_{1}$ and $\hat{v}_{1}$, (c), change over time with some circadian variation for the healthy subject. The esti- 


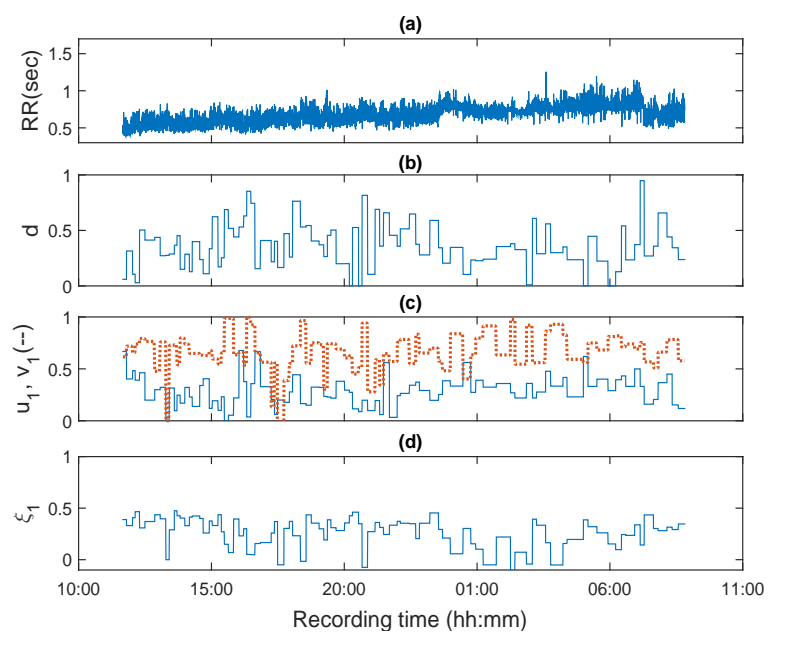

Figure 2. (a) Tachogram of healthy subject-N5, Noltisalis database, Evolution over 24 hours of $\hat{d}$ in (b), $\hat{u}_{1}(-)$ and $\hat{v}_{1}(--)$ in (c) and $\hat{\xi}_{1}$ in (d) estimated using ARFIMAEGARCH modeling.

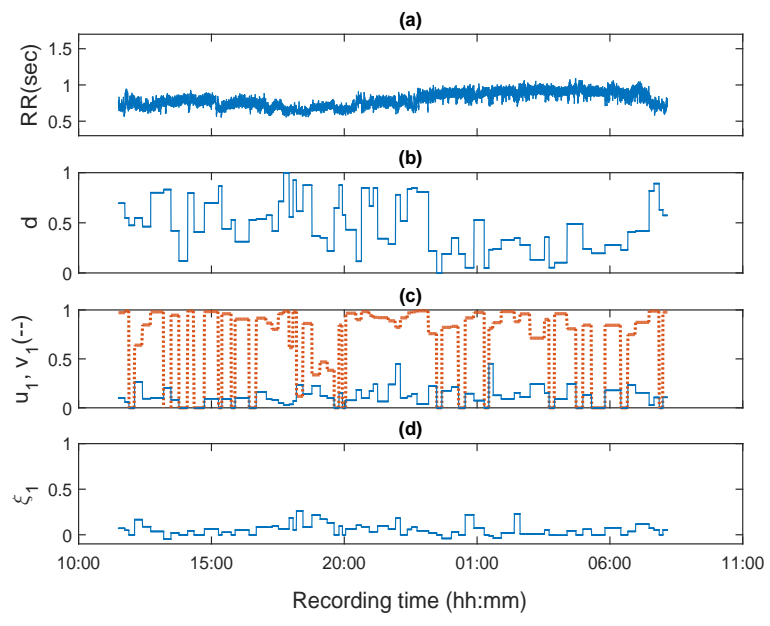

Figure 3. (a) Tachogram of patient affected by congestive heart failure-C5, Noltisalis database, Evolution over 24 hours of $\hat{d}$ in (b), $\hat{u}_{1}(-)$ and $\hat{v}_{1}(--)$ in (c) and $\hat{\xi}_{1}$ in (d) estimated using ARFIMA-EGARCH modeling.

mates $\hat{u}_{1}$ are lower for sick subjects, C5 and T3. Note that the estimated values for parameter $v_{1}$ are over 0.5 indicating some persistence in conditional variance. Moreover, the leverage parameter estimate $\hat{\xi}_{1}$, (d), changes over time and presents higher values for the healthy subject. These results are in concordance with [3,5].

The overall results for the 3 groups of patients in the database are presented during the 24 hours, 6 hours of night (Night period) and 6 hours of day (Day period), in Table 1. The results indicate that the long memory parameter $d$ increases for sick subjects, both during day and night periods with the highest values for the T group. This is consistent with [9]. The results also indicate that for the normal subjects more than $90 \%$ of the segments present volatility, while this value decreases to around $80 \%$ and $70 \%$ for the $\mathrm{C}$ and $\mathrm{T}$ groups, respectively. Additionally the volatility parameter $v_{1}$ decreases for the sick subjects both during day and night periods with lowest values and higher variability for the T group. Parameter $u_{1}$ is slightly higher for the $\mathrm{N}$ group. Finally with regard to the leverage effect the results indicate that it is much stronger in the $\mathrm{N}$ group, both in terms of percentage of segments with this effect as with regard to the value of $\xi_{1}$. The Shock Impact described in section 2.2. The impact of a negative shock is lower than that of a positive shock for the $\mathrm{N}$ group. In the diseased groups $\mathrm{C}$ and $\mathrm{T}$ the Shock Impact is negligible. It is however interesting to note that for the $\mathrm{C}$ group the impact of a negative shock is lower than that of a positive shock while for the T group is the opposite.

Statistical differences among the three groups of patients, applying the Kruskal-Wallis rank sum test and multiple comparison procedures ( $5 \%$ level of significance), are reported in Table 2. The results indicate that the long range parameter $d$ differs between the groups $\mathrm{N}$ and T, $\mathrm{C}$ and $\mathrm{T}$

Table 1. $\operatorname{ARFIMA}(p, d, 0)$-EGARCH $(1,1)$ analysis for the 3 groups of patients: healthy $(\mathrm{N})$, subjects affected by congestive heart failure (C) and transplanted (T) during 6 hours of day, 6 hours of night and 24 hour periods. The \% of segments with volatility, the $\%$ of segments with leverage and shock impact in volatility are reported. mean \pm standard deviations are presented.

\begin{tabular}{|c|c|c|c|}
\hline $\begin{array}{l}\text { Day period } \\
\text { Parameters }\end{array}$ & $\mathrm{N}$ & $\mathrm{C}$ & $\mathrm{T}$ \\
\hline$d$ & $0.45 \pm 0.06$ & $0.57 \pm 0.16$ & $0.77 \pm 0.13$ \\
\hline Seg. volatility (\%) & $92.8 \pm 9.5$ & $84.1 \pm 13.3$ & $74.4 \pm 24.4$ \\
\hline Seg. leverage $(\%)$ & $87.9 \pm 10.8$ & $57.8 \pm 23.4$ & $40.2 \pm 25.3$ \\
\hline$u_{1}$ & $0.27 \pm 0.11$ & $0.18 \pm 0.10$ & $0.17 \pm 0.15$ \\
\hline$v_{1}$ & $0.66 \pm 0.09$ & $0.63 \pm 0.21$ & $0.53 \pm 0.23$ \\
\hline$\xi_{1}$ & $0.22 \pm 0.14$ & $0.05 \pm 0.05$ & $-0.01 \pm 0.04$ \\
\hline Shock Impact & $0.50 \pm 0.21$ & $0.86 \pm 0.13$ & $1.11 \pm 0.23$ \\
\hline Night period & & & \\
\hline Parameters & $\mathrm{N}$ & $\mathrm{C}$ & $\mathrm{T}$ \\
\hline$d$ & $0.31 \pm 0.05$ & $0.36 \pm 0.16$ & $0.65 \pm 0.17$ \\
\hline Seg. volatility (\%) & $98.5 \pm 2.1$ & $89.1 \pm 5.8$ & $77.3 \pm 17.8$ \\
\hline Seg. leverage (\%) & $78.1 \pm 19.5$ & $55.7 \pm 26.4$ & $49.6 \pm 16.5$ \\
\hline$u_{1}$ & $0.29 \pm 0.08$ & $0.22 \pm 0.11$ & $0.22 \pm 0.11$ \\
\hline$v_{1}$ & $0.77 \pm 0.05$ & $0.67 \pm 0.13$ & $0.51 \pm 0.23$ \\
\hline$\xi_{1}$ & $0.12 \pm 0.08$ & $0.05 \pm 0.11$ & $-0.01 \pm 0.03$ \\
\hline Shock Impact & $0.73 \pm 0.18$ & $0.92 \pm 0.32$ & $1.13 \pm 0.18$ \\
\hline 24 hours & & & \\
\hline Parameters & $\mathrm{N}$ & $\mathrm{C}$ & $\mathrm{T}$ \\
\hline$d$ & $0.42 \pm 0.06$ & $0.50 \pm 0.14$ & $0.74 \pm 0.10$ \\
\hline Seg. volatility (\%) & $96.7 \pm 3.0$ & $84.6 \pm 10.6$ & $78.3 \pm 17.9$ \\
\hline Seg. leverage $(\%)$ & $82.8 \pm 12.5$ & $54.3 \pm 20.2$ & $44.0 \pm 12.0$ \\
\hline$u_{1}$ & $0.28 \pm 0.07$ & $0.20 \pm 0.09$ & $0.21 \pm 0.13$ \\
\hline$v_{1}$ & $0.72 \pm 0.06$ & $0.64 \pm 0.17$ & $0.56 \pm 0.20$ \\
\hline$\xi_{1}$ & $0.18 \pm 0.11$ & $0.05 \pm 0.07$ & $-0.01 \pm 0.02$ \\
\hline Shock Impact & $0.59 \pm 0.19$ & $0.89 \pm 0.18$ & $1.11 \pm 0.12$ \\
\hline
\end{tabular}




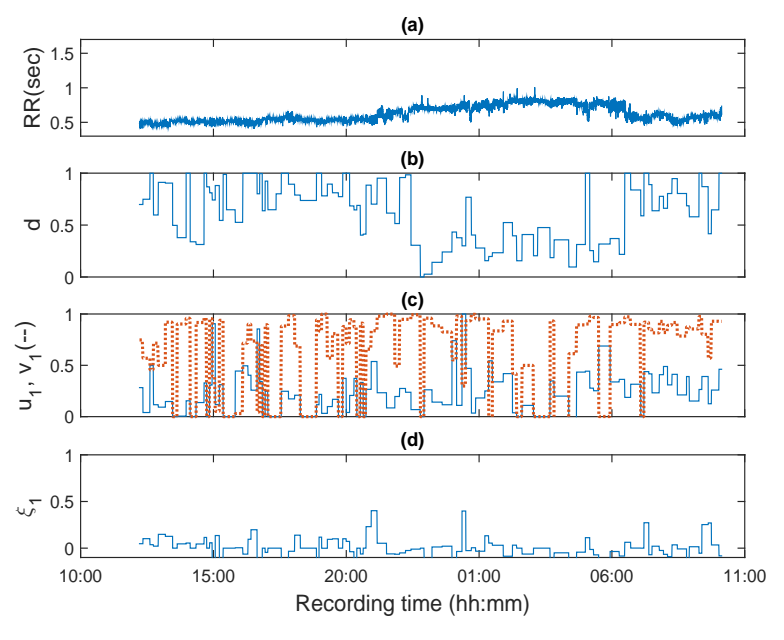

Figure 4. (a) Tachogram of heart transplanted patientT3, Noltisalis database, Evolution over 24 hours of $\hat{d}$ in (b), $\hat{u}_{1}(-)$ and $\hat{v}_{1}(--)$ in (c) and $\hat{\xi}_{1}$ in (d) estimated using ARFIMA-EGARCH modeling.

Table 2. Kruskal-Wallis rank sum test and $\%$ of correct assignments for 3 groups (healthy N, congestive heart failure $\mathrm{C}$ and transplanted T) during 6 hours of day and night periods. $\sqrt{ } \sqrt{ }(\sqrt{ }) 5 \%$ (10\%) significant differences.

\begin{tabular}{cccccc}
\hline \hline $\begin{array}{c}\text { Day period } \\
\text { Parameter }\end{array}$ & $\begin{array}{c}\text { Kruskal-Wallis } \\
p-\text { value }\end{array}$ & \multicolumn{2}{c}{ Multiple comparison } & \% Correct \\
\hline$d$ & $<0.001$ & $\ldots$ & $\sqrt{ } \sqrt{ }$ & $\sqrt{ } \sqrt{ }$ & 70.0 \\
$u_{1}$ & 0.252 & $\ldots$ & $\ldots$ & $\ldots$ & 40.0 \\
$v_{1}$ & 0.247 & $\ldots$ & $\ldots$ & $\ldots$ & 50.0 \\
$\xi_{1}$ & $<0.001$ & $\sqrt{ }$ & $\sqrt{ } \sqrt{ }$ & $\sqrt{ }$ & 70.0 \\
\hline Night period & Kruskal-Wallis & Multiple comparison & \\
Parameter & $p-$ value & N-C & N-T & C-T & \\
\hline$d$ & $<0.001$ & $\ldots$ & $\sqrt{ } \sqrt{ }$ & $\sqrt{ }$ & 60.0 \\
$u_{1}$ & 0.152 & $\ldots$ & $\ldots$ & $\ldots$ & 46.7 \\
$v_{1}$ & 0.021 & $\ldots$ & $\sqrt{ } \sqrt{ }$ & $\ldots$ & 70.0 \\
$\xi_{1}$ & 0.002 & $\sqrt{ }$ & $\sqrt{ } \sqrt{ }$ & $\ldots$ & 60.0 \\
\hline \hline
\end{tabular}

in the day and night periods. The leverage parameter $\xi_{1}$ differs among the 3 groups during the day and only between health and disease during the night. Additionally, discriminant analysis is applied to determine the ability of the parameters to distinguish among the three groups of patients. During the daytime, the parameters $d$ and $\xi_{1}$ have the highest discriminatory power, $70 \%$, among the three patient groups. During the nighttime, the parameter $v_{1}$ has the highest discriminatory power, $70 \%$. When the four parameters are combined their discriminatory power improve: during nighttime the highest value is $80 \%$ and during daytime is $70 \%$.

The above analysis indicates that ARFIMA-EGARCH models provide a set of parameters that quantify important characteristics of HRV such as long memory, time-varying volatility and asymmetry in volatility. Such parameters are promising in differentiating health and disease situations, as well as in risk stratification and autonomic nervous system dysfunction characterization, warranting further study.

\section{Acknowledgements}

Work financed by the ERDF - European Regional Development Fund through the Operational Programme for Competitiveness and Internationalisation - COMPETE 2020 Programme, project POCI-01-0145-FEDER006961, and by National Funds through the Portuguese funding agency, FCT - Fundação para a Ciência e a Tecnologia as part of projects UID/EEA/50014/2013, CIDMA UID/MAT/04106/2013 and CMUP UID/MAT/00144/2013, funded by FCT (Portugal) with national (MEC) and European structural funds through program FEDER, under partnership agreement PT2020.

\section{References}

[1] Task Force of the European Society of Cardiology and North American Society of Pacing Electrophysiology. Heart rate variability: standards of measurement, physiological interpretation and clinical use. Circulation 1996;93:1043-1065.

[2] Sassi R, Cerutti S, Lombardi F, Malik M, Huikuri H, Peng C-K, Schmidt G, Yamamoto Y. Advances in heart rate variability signal analysis: EHRA position paper. Europace 2015;17(9):1341-1353.

[3] Leite A, Rocha AP, Silva ME. Beyond long memory in heart rate variability: an approach based on fractionally integrated autoregressive moving average time series models with conditional heteroscedasticity. Chaos 2013;23:023103.

[4] Tsay RS. Analysis of Financial Time Series (2nd ed.). New Jersey: Wiley-Interscience, 2005.

[5] Leite A, Silva ME, Rocha AP. Modeling volatility in Heat Rate Variability. In: Proceedings of IEEE-EMBS International Conference, Orlando, FL USA 2016:3582-3585.

[6] Signorini MG, Sassi R, Cerutti S. Working on the NOLTISALIS Database: Measurement of nonlinear properties in heart rate variability signals. In: Proceedings of IEEEEMBS International Conference, Istanbul, Turkey (IEEE, Piscataway) 2001:547-550.

[7] Boukhan P, Oppenheim G, Taqqu MS. Theory and applications of long-range dependence. Boston: Birkhäuser, 2003.

[8] MATLAB, version 8.5 (R2015a). The MathWorks Inc., Natick, Massachusetts, 2015.

[9] Leite A, Rocha AP, Silva ME. Long memory and volatility in HRV: an ARFIMA-GARCH approach. In: Computing in Cardiology 2009;36:165-168.

Address for correspondence:

Ana Paula Rocha, aprocha@fc.up.pt.

FCUP, Univ Porto,R.Campo Alegre 687,4169-007 Porto,Portugal 